\title{
8-star-choosability of a graph with maximum average degree less than 3
}

\author{
Min Chen $^{1,2}$ \\ André Raspaud 2" \\ Weifan Wang 1 非 \\ ${ }^{1}$ Department of Mathematics, Zhejiang Normal University, Jinhua, China \\ ${ }^{2}$ LaBRI UMR CNRS 5800, Université Bordeaux I, France \\ received $4^{\text {th }}$ November 2009, revised $26^{\text {th }}$ September 2011, accepted $12^{\text {th }}$ December 2011.
}

A proper vertex coloring of a graph $G$ is called a star-coloring if there is no path on four vertices assigned to two colors. The graph $G$ is $L$-star-colorable if for a given list assignment $L$ there is a star-coloring $c$ such that $c(v) \in L(v)$. If $G$ is $L$-star-colorable for any list assignment $L$ with $|L(v)| \geq k$ for all $v \in V(G)$, then $G$ is called $k$-star-choosable. The star list chromatic number of $G$, denoted by $\chi_{s}^{l}(G)$, is the smallest integer $k$ such that $G$ is $k$-star-choosable.

In this article, we prove that every graph $G$ with maximum average degree less than 3 is 8 -star-choosable. This extends a result that planar graphs of girth at least 6 are 8-star-choosable [A. Kündgen, C. Timmons, Star coloring planar graphs from small lists, J. Graph Theory, 63(4): 324-337, 2010].

Keywords: List star coloring, maximum average degree, in-coloring, orientation.

\section{Introduction}

Only simple graphs are considered in this paper unless otherwise stated. A plane graph is a particular drawing of a planar graph in the Euclidean plane. For a graph $G$, we use $V(G), E(G),|G|$, and $\delta(G)$ to denote its vertex set, edge set, order, and minimum degree, respectively. The girth $g(G)$ of $G$ is the length of a shortest cycle in $G$.

A proper $k$-coloring of $G$ is an assignment of $k$ colors $1,2, \cdots, k$ to $V(G)$ such that adjacent vertices receive different colors. If $G$ has a proper $k$-coloring, then $G$ is said to be $k$-colorable. The chromatic number, denoted by $\chi(G)$, is the smallest integer $k$ such that $G$ is $k$-colorable. A proper vertex coloring of a graph $G$ is acyclic if there is no bicolored cycle in $G$. In other words, the graph induced by the union of every two color classes is a forest. The acyclic chromatic number, denoted by $\chi_{a}(G)$, of $G$ is the smallest integer $k$ such that $G$ has an acyclic $k$-coloring.

The notion of acyclic coloring of graphs was first introduced by Grünbaum in Grünbaum (1973). In 1979, Borodin Borodin (1979) confirmed Grünbaum's conjecture that every planar graph is acyclically

\footnotetext{
†Email: chenminezjnu.cn.

¥Email: raspaudelabri.fr

§Email: wwfez jnu.cn.
}

1365-8050 @ 2011 Discrete Mathematics and Theoretical Computer Science (DMTCS), Nancy, France 
5-colorable. This upper bound is best possible, based on two examples constructed in Grünbaum (1973) and Kostochka and Mel'nikov (1976), respectively. Grünbaum also noted that the condition that the union of any two color classes inducing a forest can be generalized to other bipartite graphs. Among other problems, he suggested requiring that the union of any pair of color classes induces a star forest, namely, a proper coloring avoiding 2-colored paths with four vertices. Formally, such coloring is called a starcoloring. The star chromatic number $\chi_{s}(G)$ is defined to be the least number of colors required to obtain a star-coloring of $G$.

Let $\mathcal{P}$ denote the family of planar graphs. By using both Borodin's acyclic 5-color theorem and Grünbaum's inequality $\chi_{s}(G) \leq \chi_{a}(G) 2^{\chi_{a}(G)-1}$, it is easy to obtain that $\chi_{s}(\mathcal{P}) \leq 80$. In 2003 , Nešetřil and Ossona de Mendez Nešetřil and Ossana de Mendez (2003) made a big step by showing that $\chi_{s}(\mathcal{P}) \leq 30$. Then, Albertson et al. Albertson et al. (2004) further decreased this upper bound to 20 and gave a lower bound by showing an example of a planar graph $H$ which needs at least 10 colors to star color. It follows that $10 \leq \chi_{s}(\mathcal{P}) \leq 20$. Moreover, they observed that the graph $C_{n}^{+}$(an $n$-cycle with a leaf vertex added to each vertex of the cycle) has star chromatic number 4 when $n$ is not divisible by 3 . Additionally, they proved that planar graphs of girth at least 5 (resp. 7) can be star colored with 16 (resp. 9) colors. Other star-coloring results are provided in Bu et al. (2009); Fertin et al. (2001) and Timmons (2007).

We say that $G$ is $L$-star-colorable if for a given list assignment $L$ there is a star-coloring $c$ such that $c(v) \in L(v)$. If $G$ is $L$-star-colorable for any list assignment $L$ with $|L(v)| \geq k$ for all $v \in V(G)$, then $G$ is $k$-star-choosable. The star list chromatic number, or star choice number, denoted by $\chi_{s}^{l}(G)$, of $G$ is the smallest integer $k$ such that $G$ is $k$-star-choosable.

Recently, $L$-star-coloring has been investigated by some authors. Kierstead, Kündgen and Timmons Kierstead et al. (2009) showed that bipartite planar graphs are 14-star-choosable, and gave an example of a bipartite planar graph that requires 8 colors to star color. Chen, Raspaud and Wang Chen et al. (2011) showed that if $G$ is a planar subcubic graph, then (1) $\chi_{s}^{l}(G) \leq 6$ if $g(G) \geq 3$; (2) $\chi_{s}^{l}(G) \leq 5$ if $g(G) \geq 8$; and (3) $\chi_{s}^{l}(G) \leq 4$ if $g(G) \geq 12$.

The maximum average degree $\operatorname{mad}(G)$ of a graph $G$ is defined as:

$$
\operatorname{mad}(G)=\max \left\{\frac{2|E(H)|}{|V(H)|}: H \subseteq G\right\} .
$$

Kündgen and Timmons Kündgen and Timmons (2010) proved the following.

Theorem 1 (Kündgen and Timmons (2010)) Let $G$ be a graph.

(1) If $\operatorname{mad}(G)<\frac{8}{3}$, then $\chi_{s}^{l}(G) \leq 6$.

(2) If $\operatorname{mad}(G)<\frac{14}{5}$, then $\chi_{s}^{l}(G) \leq 7$.

(3) If $G$ is planar and $g(G) \geq 6$, then $\chi_{s}^{l}(G) \leq 8$.

It is well known that a planar graph $G$ with girth $g(G)$ satisfies $\operatorname{mad}(G)<\frac{2 g(G)}{g(G)-2}$. Using this fact and (1) and (2) in Theorem 1, we immediately deduce that for a planar graph $G$, we have $\chi_{s}^{l}(G) \leq 6$ if $g(G) \geq 8$, and $\chi_{s}^{l}(G) \leq 7$ if $g(G) \geq 7$.

The purpose of this paper is to extend the result (3) in Theorem 1 to graphs with maximum average degree less than 3 . More precisely, we will prove the following:

Theorem 2 Every graph $G$ with $\operatorname{mad}(G)<3$ is 8-star-choosable. 


\section{Preliminaries}

For simplicity, we use $i^{+}$to denote a number of at least $i$. A $k$-vertex, $k^{+}$-vertex, or $k^{-}$-vertex is a vertex of degree $k$, at least $k$, or at most $k$. A $k(d)$-vertex is a $k$-vertex adjacent to $d 2$-vertices. For $v \in V(G)$, let $n_{2}(v)$ denote the number of 2-vertices adjacent to $v$, and $N_{G}(v)$ denote the set of neighbors of $v$ in $G$. A walk of $G$ is a non-empty alternating sequence of vertices and edges denoted by $W=v_{1} e_{1} v_{2} e_{2} \cdots e_{k-1} v_{k}$, where $e_{i}=v_{i} v_{i+1}$ for each $1 \leq i \leq k-1$. If all the vertices of a walk $v_{1} e_{1} v_{2} e_{2} \cdots e_{k-1} v_{k}$ are mutually distinct, then we call such a walk a path, simply denoted by $P=v_{1} v_{2} \cdots v_{k-1} v_{k}$. We say that $v_{1}, v_{k}$ are the endpoints of $P$ and $v_{2}, \cdots, v_{k-1}$ are internal vertices of $P$. For $S \subseteq V(G)$, let $G-S$ denote the subgraph of $G$ obtained by deleting the vertices in $S$ and all edges incident to some vertices in $S$. Let $G[S]$ denote the subgraph of $G$ induced by $S$.

Let $x y \in E(G)$. We use $x \rightarrow y$ to denote that the edge $x y$ is oriented from $x$ to $y$. For a simple undirected graph $G$, an orientation of $G$ is obtained by assigning a direction to each edge, denoted by $\vec{G}$. For $v \in V(\vec{G})$, we define the outdegree vertices set of $v$ by $D_{\vec{G}}^{+}(v)=\left\{u \mid u \in N_{\vec{G}}(v)\right.$ and $\left.v \rightarrow u\right\}$. A special orientation $\vec{G}$ of $G$ is an orientation in which each vertex $v$ satisfies $\left|D_{\vec{G}}^{+}(v)\right| \leq 2$.

In order to study the star chromatic number of graphs, Albertson et al. Albertson et al. (2004) introduced the following useful concept.

A proper coloring of an oriented graph $G$ is called an in-coloring if for every 2-colored $P_{3}$ on three vertices in $G$, the edges are directed towards the middle vertex. A coloring of $G$ is an in-coloring if it is an in-coloring of some orientation of $G$. An $L$-in-coloring of $G$ is an in-coloring of $G$ such that the colors are chosen from the lists assigned to each vertex. We say that a graph $G$ with a given orientation is $k$-in-choosable if it is $L$-in-colorable for every list assignment $L$ with $|L(v)| \geq k$ for all $v \in V(G 0)$.

Though the proof of the following Lemma 1 is very similar to that of Lemma 3.2 in Albertson et al. (2004), we like to write, for completeness, its details.

Lemma 1 An L-coloring of a graph $G$ is an L-star-coloring if and only if it is an L-in-coloring of some orientation of $G$.

Proof. Given an $L$-star-coloring, we can construct an orientation by directing the edges towards the center of the star in each star-forest corresponding to the union of two color classes.

Conversely, consider an $L$-in-coloring of $\vec{G}$, an orientation of $G$. Let $P_{4}=u v w z$ be any path on four vertices in $G$. We may assume that the edge $v w$ is directed towards $w$ in $\vec{G}$. For the given coloring to be an $L$-in-coloring at $v$, we must have three different colors on $u, v$ and $w$.

So, in order to control the number of colors used in an in-coloring, it is useful to bound the maximum outdegree of the orientation $\vec{G}$. In 1981, Tarsi Tarsi $(1981)$ observed the fact that a graph has an orientation with maximum outdegree at most $d$ if and only if $\operatorname{mad}(G) \leq 2 d$. This implies that every graph with $\operatorname{mad}(G)<3$ has an orientation with maximum outdegree at most 2 . Therefore, to obtain our Theorem 2 . by Lemma 1 , we only need to prove the following Theorem 3 .

Theorem 3 Every graph $G$ with $\operatorname{mad}(G)<3$ has an orientation of maximum outdegree at most 2 that makes the graph 8-in-choosable.

The following section is dedicated to the proof of Theorem 3 . For all figures depicted in Section 3, a vertex is represented by a solid point when all of its incident edges are drawn; otherwise it is represented by a hollow point. 


\section{Proof of Theorem 3}

In what follows, let $L$ be an uncolorable list assignment of $G$ with $|L(v)|=8$ for all $v \in V(G)$. Suppose that $G$ is a counterexample with the least number of vertices to Theorem 3 Thus $G$ is connected. Moreover, any subgraph $H$ with $|H|<|G|$ admits an $L$-in-coloring of some special orientation $\vec{H}$. We first discuss some properties of $G$, then use discharging technique to derive a contradiction.

By the definition of maximum average degree and Tarsi's observation, we first note the following statement.

Observation 1 Every subgraph $H \subseteq G$ admits a special orientation.

So, in the following, we always admit a special orientation $\vec{H}$ of $H$. Moreover, for $v \in V(\vec{H})$, define $N_{\vec{H}}^{*}(v)=D_{\vec{H}}^{+}(v) \cup\{v\}$. It is obvious that $\left|N_{\vec{G}}^{*}(v)\right| \leq 3$. For simplicity, we write $N^{*}(v)$ for $N_{\vec{H}}^{*}(v)$. We further use $S(v)$ to denote the set of vertices whose coloring is forbidden on $v$ by the definition of $L$-in-coloring when we are about to color $v$.

In the proofs below, in the subgraph in question the vertices will be taken in an appropriate order in such a way that when we color a vertex $v$, in the subgraph which is colored before $v$, the number of vertices putting a constraint to $v$ is smaller than the list size of $v$, and therefore each vertex can get a color from its list in this order.

Claim 1 G contains no 1-vertex.

Proof. Suppose that $x$ is a 1-vertex of $G$ and $y$ is the neighbor of $x$. Let $H=G-\{x\}$. By the minimality of $G, H$ admits an $L$-in-coloring $c$ of some special orientation $\vec{H}$. We orient the edge $x y$ from $x$ to $y$ to establish an orientation $\vec{G}$ of $G$. Clearly, the resulting orientation $\vec{G}$ is special. Now, we assign a color to $x$ in $L(x)$, different from the colors of the vertices in $N^{*}(y)$. It is easy to see that the color for $x$ is proper and thus we extend $c$ to $G$, which is a contradiction.

In the proofs of Claims 2 to 8 , we use $\mathcal{B}$ to denote the set of all solid vertices, depicted in Fig. 1 to Fig. 6. Let $H=G-\mathcal{B}$. By the minimality of $G, H$ admits an $L$-in-coloring $c$ of some special orientation $\vec{H}$. We give an orientation of $G[\mathcal{B}]$ and those edges between $V(H)$ and $\mathcal{B}$ such that the resulting orientation $\vec{G}$ is special. Then we extend $c$ to $\mathcal{B}$ to obtain an $L$-in-coloring of $\vec{G}$, which contradicts the choice of $G$.

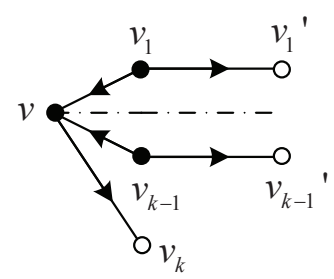

Fig. 1: $v$ is a $k(k-1)$-vertex.

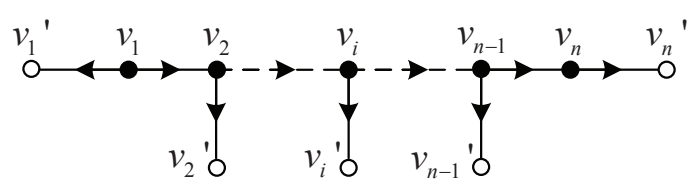

Fig. 2: A good path $P=v_{1} v_{2} \cdots v_{n}$.

Claim 2 G contains no $k(k-1)$-vertex for any $2 \leq k \leq 5$.

Proof. Assume to the contrary that $v$ is a $k(k-1)$-vertex with $2 \leq k \leq 5$. Let $v_{1}, \cdots, v_{k}$ denote the neighbors of $v$. Without loss of generality, assume that $d\left(v_{i}\right)=2$ for all $i \in\{1, \cdots, k-1\}$ and $d\left(v_{k}\right) \geq 2$. For each $i \in\{1, \cdots, k-1\}$, let $v_{i}^{\prime}$ be the other neighbor of $v_{i}$ different from $v$. 
Let $\mathcal{B}=\left\{v, v_{1}, \cdots, v_{k-1}\right\}$ and $H=G-\mathcal{B}$. By the minimality of $G, H$ has an $L$-in-coloring $c$ of some special orientation $\vec{H}$. We construct an orientation for the edge set $E(G[\mathcal{B}])$ and those edges between $V(H)$ and $\mathcal{B}$, as shown in Fig. 1. The resulting orientation $\vec{G}$ is also special. Notice that $\left|N^{*}(u)\right| \leq 3$ for each $u \in\left\{v_{1}^{\prime}, \cdots, v_{k-1}^{\prime}, v_{k}\right\}$. Based on $c$, we can color $v, v_{1}, \cdots, v_{k-1}$, successively, because

- $S(v)=N^{*}\left(v_{k}\right) \cup\left\{v_{1}^{\prime}, \cdots, v_{k-1}^{\prime}\right\}$;

- $S\left(v_{i}\right)=N^{*}\left(v_{i}^{\prime}\right) \cup\left\{v, v_{k}\right\}$, for each $i \in\{1, \cdots, k-1\}$.

Obviously, for each vertex $x \in \mathcal{B}$ we have $|S(x)| \leq 3+(k-1)=k+2 \leq 7$ because $2 \leq k \leq 5$. So the resulting coloring is an $L$-in-coloring of $\vec{G}$, which is a contradiction.

Assume that $P=v_{1} v_{2} \cdots v_{n}$ is an induced path with $n \geq 3$ and all internal vertices are 3 -vertices. If $d\left(v_{1}\right)=d\left(v_{n}\right)=2$ then $P$ is called a good path. If $d\left(v_{1}\right)=2$ and $d\left(v_{n}\right) \geq 4$ then $P$ is called a bad path. If $d\left(v_{1}\right)=2$ and $d\left(v_{n}\right)=3$ then $P$ is called a terrible path. For simplicity, we use $P\left(v_{1} \rightarrow v_{n}\right)$ to denote an orientation for the edge set $E(P)$ in such a way that $v_{i} \rightarrow v_{i+1}$ for each $i \in\{1, \cdots, n-1\}$.

Claim 3 There is no good path in $G$.

Proof. Assume to the contrary that there exists a good path $P=v_{1} v_{2} \cdots v_{n}$ with $n \geq 3$ in $G$. By definition, $v_{1}, v_{n}$ are both 2 -vertices and the remaining vertices are all 3 -vertices. Since $P$ is an induced path, for each vertex $v_{i} \in V(P)$, we may let $v_{i}^{\prime}$ be the other neighbor of $v_{i}$ which is not on $P$.

Let $\mathcal{B}=\left\{v_{1}, \cdots, v_{n}\right\}$ and $H=G-\mathcal{B}$. By the minimality of $G, H$ has an $L$-in-coloring $c$ of some special orientation $\vec{H}$. We define an orientation for the edge set $E(G[\mathcal{B}]) \cup\left\{v_{1} v_{1}^{\prime}, \cdots, v_{i} v_{i}^{\prime}, \cdots, v_{n} v_{n}^{\prime}\right\}$ in the following way: $P\left(v_{1} \rightarrow v_{n}\right)$ and $v_{j} \rightarrow v_{j}^{\prime}$ for each $j \in\{1, \cdots, n\}$, as depicted in Fig. 2. The resulting orientation $\vec{G}$ is special. We can color $v_{1}, v_{2}, \cdots, v_{n}$, successively, because

- $S\left(v_{1}\right)=N^{*}\left(v_{1}^{\prime}\right) \cup\left\{v_{2}^{\prime}\right\}$;

- $S\left(v_{2}\right)=N^{*}\left(v_{2}^{\prime}\right) \cup\left\{v_{1}, v_{1}^{\prime}, v_{3}^{\prime}\right\}$

- $S\left(v_{i}\right)=N^{*}\left(v_{i}^{\prime}\right) \cup\left\{v_{i-1}, v_{i-2}, v_{i-1}^{\prime}, v_{i+1}^{\prime}\right\}$, for each $i \in\{3, \cdots n-1\}$;

- $S\left(v_{n}\right)=N^{*}\left(v_{n}^{\prime}\right) \cup\left\{v_{n-1}, v_{n-2}, v_{n-1}^{\prime}\right\}$.

Since $|S(v)| \leq 7$ for each vertex $v \in \mathcal{B}$, the obtained coloring is an $L$-in-coloring of $G$, which is a contradiction.

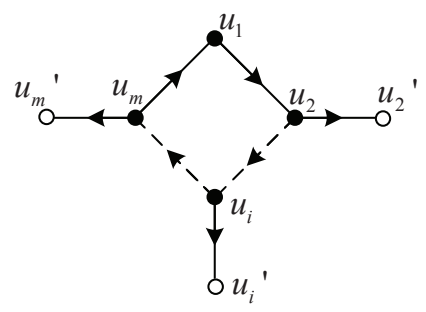

Fig. 3: A good cycle $C=u_{1} u_{2} \cdots u_{m} u_{1}$.

A cycle $C$ is called good if $C$ is formed from a good path $P=v_{1} v_{2} \cdots v_{n}$ by identifying 2-vertices $v_{1}$ and $v_{n}$.

Claim 4 There is no good cycle in $G$. 
Proof. Suppose to the contrary that $C=u_{1} u_{2} \cdots u_{m} u_{1}$ is a good cycle such that $d\left(u_{1}\right)=2$ and $d\left(u_{i}\right)=3$ for all $i \in\{2, \cdots, m\}$. Notice that $m \geq 3$. Since $C$ is formed from a good path which is also an induced path, we may let $u_{i}^{\prime}$ be the third neighbor of $u_{i}$ that is not on $C$, for each $i \in\{2, \cdots, m\}$.

Let $\mathcal{B}=\left\{u_{1}, \cdots, u_{m}\right\}$ and $H=G-\mathcal{B}$. By the choice of $G, H$ admits an $L$-in-coloring $c$ of a special orientation $\vec{H}$. We define an orientation for the edge set $E(G[\mathcal{B}]) \cup\left\{u_{2} u_{2}^{\prime}, \cdots, u_{i} u_{i}^{\prime}, \cdots, u_{m} u_{m}^{\prime}\right\}$ in the following way: for each $j \in\{2, \cdots, m-1\}$, set $u_{j} \rightarrow u_{j+1}, u_{j} \rightarrow u_{j}^{\prime}$; we further set $u_{1} \rightarrow u_{2}, u_{m} \rightarrow u_{1}$ and $u_{m} \rightarrow u_{m}^{\prime}$, see Fig. 3. We notice that the resulting orientation $\vec{G}$ is also special. Based on $c$, we can color $u_{2}, u_{3}, \cdots, u_{m}, u_{1}$, successively, because

- $S\left(u_{2}\right)=N^{*}\left(u_{2}^{\prime}\right) \cup\left\{u_{3}^{\prime}\right\}$

- $S\left(u_{i}\right)=N^{*}\left(u_{i}^{\prime}\right) \cup\left\{u_{i-1}, u_{i-2}, u_{i-1}^{\prime}, u_{i+1}^{\prime}\right\}$, for each $i \in\{3, \cdots, m-1\}$;

- $S\left(u_{m}\right)=N^{*}\left(u_{m}^{\prime}\right) \cup\left\{u_{2}, u_{m-1}, u_{m-2}, u_{m-1}^{\prime}\right\}$;

- $S\left(u_{1}\right)=\left\{u_{2}, u_{2}^{\prime}, u_{3}, u_{m}, u_{m}^{\prime}, u_{m-1}\right\}$.

Since $|S(v)| \leq 7$ for each vertex $v \in \mathcal{B}$, the resultant coloring is an $L$-in-coloring of $G$.

A cycle $C$ is called light if every vertex is of degree 3. A chordless light cycle is a light cycle that is chordless. Suppose that $C=v_{1} v_{2} \cdots v_{n} v_{1}$ is a chordless light cycle. If there exists a terrible path $P$ connecting one vertex in $C$, say $v_{1}$, such that $V(P) \cap V(C)=\left\{v_{1}\right\}$, then $C$ is called a removable cycle, where $v_{1}$ is called a heavy 3 -vertex of $C$.

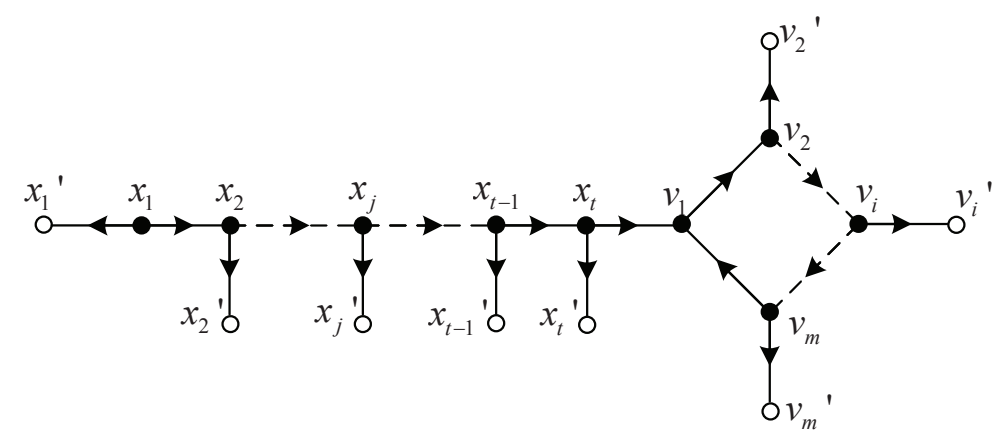

Fig. 4: A removable cycle $C=v_{1} v_{2} \cdots v_{m} v_{1}$ with a heavy 3-vertex $v_{1}$.

Claim 5 There is no removable cycle in $G$.

Proof. Suppose to the contrary that there exists a removable cycle $C=v_{1} v_{2} \cdots v_{m} v_{1}$ with a heavy 3vertex $v_{1}$ such that $P=x_{1} \cdots x_{t} v_{1}$ is a terrible path. Namely, $x_{1}$ is a 2-vertex and the remaining other vertices of $P$ are 3-vertices such that $V(P) \cap V(C)=\left\{v_{1}\right\}$. For each $i \in\{2, \cdots, m\}$, let $v_{i}^{\prime}$ be the other neighbor of $v_{i}$ not on $C$. Since $P$ is an induced path, we further let $x_{j}^{\prime}$ be the other neighbor of $x_{j}$ not on $P$ for each $j \in\{1, \cdots, t\}$. In the following, denote $A_{1}=\left\{x_{1}, \cdots, x_{t}\right\}$ and $A_{2}=\left\{v_{2}, \cdots, v_{m}\right\}$. We have to consider the following two cases.

Case $1 w z \notin E(G)$ for all $w \in A_{1}$ and $z \in A_{2}$.

It means that the third neighbor of $x_{j}$ is not in $C$, for all $j$. Let $\mathcal{B}=V(C) \cup V(P)$ and $H=G-\mathcal{B}$. By the choice of $G, H$ admits an $L$-in-coloring $c$ of a special orientation $\vec{H}$. We define an orientation for the 
edge set $E(G[\mathcal{B}])$ and those edges between $V(H)$ and $\mathcal{B}$, as shown in Fig. 4. We note that $\vec{G}$ is a special orientation. So we can color $v_{2}, \cdots, v_{m}, v_{1}, x_{t}, \cdots, x_{1}$, successively, because

- $S\left(v_{2}\right)=N^{*}\left(v_{2}^{\prime}\right) \cup\left\{v_{3}^{\prime}\right\}$;

- $S\left(v_{i}\right)=N^{*}\left(v_{i}^{\prime}\right) \cup\left\{v_{i-1}, v_{i-2}, v_{i-1}^{\prime}, v_{i+1}^{\prime}\right\}$, for each $i \in\{3, \cdots, m-1\}$;

- $S\left(v_{m}\right)=N^{*}\left(v_{m}^{\prime}\right) \cup\left\{v_{m-1}, v_{m-2}, v_{m-1}^{\prime}, v_{2}\right\}$;

- $S\left(v_{1}\right)=\left\{v_{2}, v_{2}^{\prime}, v_{3}, v_{m}, v_{m}^{\prime}, v_{m-1}\right\}$;

- $S\left(x_{t}\right)=N^{*}\left(x_{t}^{\prime}\right) \cup\left\{x_{t-1}^{\prime}, v_{1}, v_{2}\right\}$;

- $S\left(x_{t-1}\right)=N^{*}\left(x_{t-1}^{\prime}\right) \cup\left\{x_{t-2}^{\prime}, x_{t}, x_{t}^{\prime}, v_{1}\right\}$;

- $S\left(x_{j}\right)=N^{*}\left(x_{j}^{\prime}\right) \cup\left\{x_{j-1}^{\prime}, x_{j+1}, x_{j+2}, x_{j+1}^{\prime}\right\}$, for each $j \in\{t-2, \cdots, 2\}$;

- $S\left(x_{1}\right)=N^{*}\left(x_{1}^{\prime}\right) \cup\left\{x_{2}, x_{2}^{\prime}, x_{3}\right\}$.

Case $2 w z \in E(G)$, for some $w \in A_{1}$ and $z \in A_{2}$.

Case 2.1 $w=x_{1}$ and $z \in A_{2}$.

This means that $x_{1} v_{s} \in E(G)$, where $s \in\{2,3, \cdots, m\}$. If none of $v_{s+1}, \cdots, v_{m}$ is adjacent to $x_{j}$ for some fixed $j \in\{2, \cdots, t\}$, then $x_{1} x_{2} \cdots x_{t} v_{1} v_{m} v_{m-1} \cdots v_{s} x_{1}$ is a good cycle, which contradicts Claim 4 Otherwise, we may suppose that $x_{j} v_{k} \in E(G)$ for some fixed $k \in\{s+1, \cdots, m\}$ such that there is no edge between $\left\{x_{2}, x_{3} \cdots, x_{j-1}\right\}$ and $\left\{v_{s+1}, v_{s+2}, \cdots, v_{k-1}\right\}$. However, a good cycle $x_{1} x_{2} \cdots x_{j} v_{k} v_{k-1} \cdots v_{s} x_{1}$ is established, contradicting Claim 4 .

Case $2.2 w \in\left\{x_{2}, x_{3}, \cdots, x_{t}\right\}$ and $z \in A_{2}$.

We may suppose that $x_{j} v_{s} \in E(G)$ for some fixed $s \in\{2, \cdots, m\}$ such that there is no edge between $\left\{x_{2}, x_{3} \cdots, x_{j-1}\right\}$ and $V(C)-\left\{v_{1}\right\}$. If $x_{l} v_{q} \notin E(G)$ for all $l \in\{j+1, j+2, \cdots, t\}$ and $q \in\{s+1, s+$ $2, \cdots, m\}$, then a removable cycle $x_{j} x_{j+1} \cdots x_{t} v_{1} v_{m} v_{m-1} \cdots v_{s} x_{j}$ with a heavy 3 -vertex $x_{j}$ is formed and then the proof is reduced to the former Case 1. Otherwise, we may suppose that $x_{k} v_{q} \in E(G)$ for some fixed $q \in\{s+1, s+2, \cdots, m\}$ such that there is no edge between $\left\{x_{j+1}, x_{j+2}, \cdots, x_{k-1}\right\}$ and $\left\{v_{s+1}, v_{s+2}, \cdots, v_{q-1}\right\}$. However, a removable cycle $x_{j} x_{j+1} \cdots x_{k} v_{q} v_{q-1} \cdots v_{s} x_{j}$ with a heavy 3 -vertex $x_{j}$ is constructed which has been settled in the previous Case 1 .

Suppose that $P=v_{1} v_{2} \cdots v_{n}$ is a bad path such that $d\left(v_{1}\right)=2, d\left(v_{n}\right) \geq 4$, and $d\left(v_{i}\right)=3$ for all $i \in\{2, \cdots, n-1\}$. We say that $v_{n}$ is a sponsor of $v_{2}$ and $v_{2}$ is a target of $v_{n}$. Moreover, let $\mathcal{T}\left(v_{n}\right)$ denote the set of targets of $v_{n}$ and let $\mathcal{S P}\left(v_{2}\right)$ denote the set of sponsors of $v_{2}$.

Claim 6 For each $4^{+}$-vertex $v$, we have $|\mathcal{T}(v)| \leq d(v)-n_{2}(v)$.

Proof. Let $x_{1}$ be a $3^{+}$-vertex adjacent to $v$. It suffices to show that there is at most one bad path starting from edge $v x_{1}$. If $d\left(x_{1}\right) \geq 4$, then $v x_{1}$ is not a bad path and thus we are done. Otherwise, we may suppose that $P=v x_{1} \cdots x_{m}$ is a bad path with a target $x_{m-1}$ such that $d\left(x_{m}\right)=2$ and $d\left(x_{i}\right)=3$ for all $i=1, \cdots, m-1$. Next, we are going to show that there is no other bad path starting from edge $v x_{1}$ and thus conclude the proof of Claim 6 .

Without loss of generality, assume that $P \neq P^{\prime}=v x_{1} \cdots x_{i} x_{i+1}^{\prime} \cdots x_{s-1}^{\prime} x_{s}^{\prime}$ is a bad path with a target $x_{s-1}^{\prime}$ of $v$. So $d\left(x_{s}^{\prime}\right)=2$ and $d\left(x_{k}^{\prime}\right)=3$ for all $k \in\{i+1, \cdots, s-1\}$. Let $B_{1}=\left\{x_{i+1}^{\prime}, x_{i+2}^{\prime}, \cdots, x_{s}^{\prime}\right\}$ and $B_{2}=\left\{x_{i+1}, x_{i+2}, \cdots, x_{m}\right\}$. The proof is divided into the two cases below.

Case $1 w z \notin E(G)$ for all $w \in B_{1}$ and $z \in B_{2}$. 
This implies that $B_{1} \cap B_{2}=\emptyset$. It is easy to observe that a good path $x_{m} x_{m-1} \cdots x_{i+1} x_{i} x_{i+1}^{\prime}$ $x_{i+2}^{\prime} \cdots x_{s}^{\prime}$ is established. This contradicts Claim 3 .

Case $2 w z \in E(G)$, where $w \in B_{1}$ and $z \in B_{2}$.

By symmetry, we only need to consider the following two possibilities.

Case 2.1 $w \in\left\{x_{i+1}^{\prime}, x_{i+2}^{\prime}, \cdots, x_{s-1}^{\prime}\right\}$ and $z \in\left\{x_{i+1}, x_{i+2}, \cdots, x_{m-1}\right\}$.

Denote $z=x_{k}$ for some fixed $k \in\{i+1, i+2, \cdots, m-1\}$. We may assume $x_{j}^{\prime}=w$ such that $x_{j}^{\prime} x_{k} \in E(G)$ and $x_{j}^{\prime}$ is the nearest 3 -vertex to $x_{i}$ on $P^{\prime}$. In other words, there are no edges between $\left\{x_{i+1}^{\prime}, x_{i+2}^{\prime}, \cdots, x_{j-1}\right\}$ and $\left\{x_{i+1}, x_{i+2}, \cdots, x_{k-1}\right\}$. It is obvious that $x_{i} x_{i+1} \cdots x_{k} x_{j}^{\prime} x_{j-1}^{\prime} \cdots x_{i+1}^{\prime} x_{i}$ is a chordless light cycle with a heavy 3 -vertex $x_{k}$. Such kind of cycle is removable, which is a contradiction to Claim 5

Case 2.2 $w \in\left\{x_{i+1}^{\prime}, x_{i+2}^{\prime}, \cdots, x_{s-1}^{\prime}\right\}$ and $z=x_{m}$.

Denote $w=x_{j}$, where $j \in\{i+1, \cdots, s-1\}$. Obviously, $x_{m} x_{m-1} \cdots x_{i+1} x_{i} x_{i+1}^{\prime} \cdots x_{j}^{\prime} x_{m}$ is a good cycle, which is impossible by Claim 4

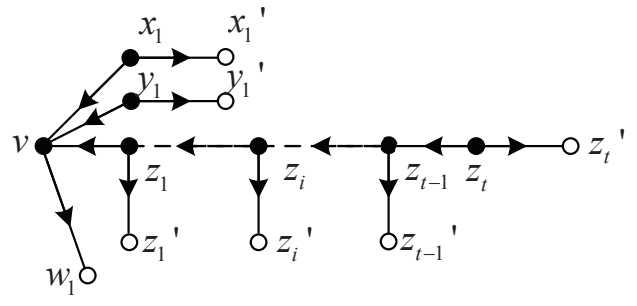

(1) $z_{t} \notin\left\{x_{1}, y_{1}\right\}$.

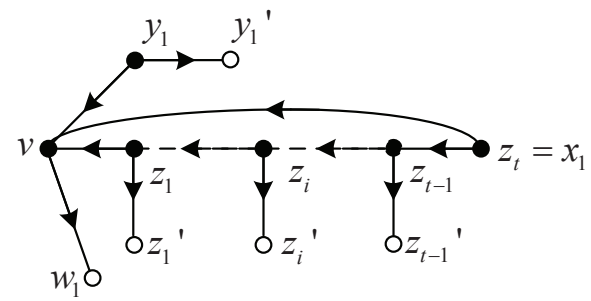

(2) $z_{t}=y_{1}$.

Fig. 5: $v$ is a 4(2)-vertex with a target $z_{t-1}$.

Claim 7 If $v$ is a $4(2)$-vertex, then $|\mathcal{T}(v)|=0$.

Proof. Let $v$ be a 4(2)-vertex with four neighbors $x_{1}, y_{1}, w_{1}, z_{1}$ such that $d\left(x_{1}\right)=d\left(y_{1}\right)=2$ and $d\left(z_{1}\right), d\left(w_{1}\right) \geq 3$. Suppose to the contrary that $|\mathcal{T}(v)| \geq 1$. We further suppose that $P=v z_{1} \cdots z_{t}$ is a bad path connecting $v$ and $v$ 's target $z_{t-1}$. Let $N_{G}\left(x_{1}\right)=\left\{v, x_{1}^{\prime}\right\}$ and $N_{G}\left(y_{1}\right)=\left\{v, y_{1}^{\prime}\right\}$. For each $k \in\{1, \cdots, t\}$, let $z_{k}^{\prime}$ be the other neighbor of $z_{k}$ that is not on $P$. Obviously, $x_{1} \neq y_{1}$. Let $\mathcal{B}=V(P) \cup\left\{x_{1}, y_{1}\right\}$ and $H=G-\mathcal{B}$. Let $c$ denote an $L$-in-coloring of $H$ for its special orientation $\vec{H}$. By the symmetry of $G$, we only need to consider the two cases below.

Case $1 z_{t} \notin\left\{x_{1}, y_{1}\right\}$.

We define an orientation for the edge set $E(G[\mathcal{B}])$ and the edges between $V(H)$ and $\mathcal{B}$, as depicted in Fig. 5(1). The resulting orientation of $\vec{G}$ is a special orientation. Based on $c$, we can color $v, x_{1}, y_{1}, z_{1}, \cdots, z_{t}$, successively, because

- $S(v)=N^{*}\left(w_{1}\right) \cup\left\{x_{1}^{\prime}, y_{1}^{\prime}, z_{1}^{\prime}\right\}$;

- $S\left(x_{1}\right)=N^{*}\left(x_{1}^{\prime}\right) \cup\left\{v, w_{1}\right\}$; 
- $S\left(y_{1}\right)=N^{*}\left(y_{1}^{\prime}\right) \cup\left\{v, w_{1}\right\}$;

- $S\left(z_{1}\right)=N^{*}\left(z_{1}^{\prime}\right) \cup\left\{v, w_{1}, z_{2}^{\prime}\right\}$;

- $S\left(z_{2}\right)=N^{*}\left(z_{2}^{\prime}\right) \cup\left\{z_{1}, z_{1}^{\prime}, v, z_{3}^{\prime}\right\}$

- $S\left(z_{i}\right)=N^{*}\left(z_{i}^{\prime}\right) \cup\left\{z_{i-1}, z_{i-2}, z_{i-1}^{\prime}, z_{i+1}^{\prime}\right\}$, for each $i \in\{3, \cdots, t-1\}$;

- $S\left(z_{t}\right)=N^{*}\left(z_{t}^{\prime}\right) \cup\left\{z_{t-1}, z_{t-1}^{\prime}, z_{t-2}\right\}$.

Case $2 z_{t}=y_{1}$.

We define an orientation for the edge set $E(G[\mathcal{B}])$ and those edges between $V(H)$ and $\mathcal{B}$, as shown in Fig. 5(2). We observe that the resulting orientation of $\vec{G}$ is special. Based on $c$, we may color $v, x_{1}, z_{1}, \cdots, z_{t}$, successively, because

- $S(v)=N^{*}\left(w_{1}\right) \cup\left\{x_{1}^{\prime}, z_{1}^{\prime}\right\}$;

- $S\left(x_{1}\right)=N^{*}\left(x_{1}^{\prime}\right) \cup\left\{v, w_{1}\right\}$;

- $S\left(z_{1}\right)=N^{*}\left(z_{1}^{\prime}\right) \cup\left\{v, w_{1}, z_{2}^{\prime}\right\}$;

- $S\left(z_{2}\right)=N^{*}\left(z_{2}^{\prime}\right) \cup\left\{z_{1}, z_{1}^{\prime}, v, z_{3}^{\prime}\right\}$

- $S\left(z_{i}\right)=N^{*}\left(z_{i}^{\prime}\right) \cup\left\{z_{i-1}, z_{i-2}, z_{i-1}^{\prime}, z_{i+1}^{\prime}\right\}$, for each $i \in\{3, \cdots, t-1\}$;

- $S\left(z_{t}\right)=\left\{v, w_{1}, z_{t-1}, z_{t-1}^{\prime}, z_{t-2}\right\}$.

Therefore, we have completed the proof of Claim 7

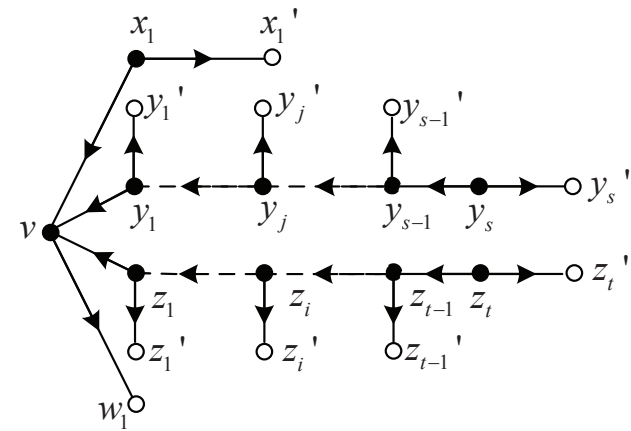

Fig. $6(1): x_{1} \neq y_{s} \neq z_{t} \neq x_{1}$.

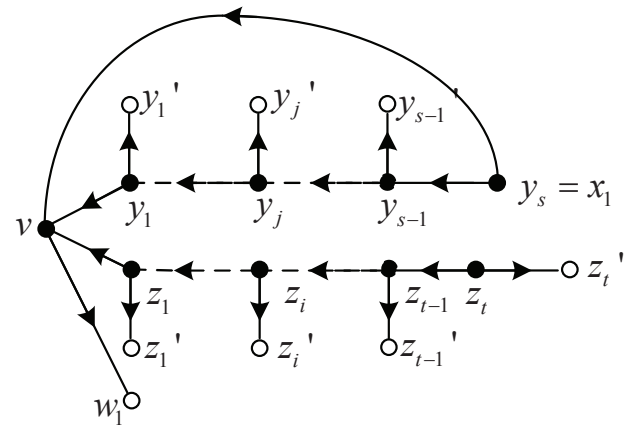

Fig. $6(2): x_{1}=y_{s} \neq z_{t}$.

Claim 8 If $v$ is a $4(1)$-vertex, then $|\mathcal{T}(v)| \leq 1$.

Proof. Let $v$ be a 4(1)-vertex with four neighbors $x_{1}, y_{1}, w_{1}, z_{1}$ such that $x_{1}$ is a 2-vertex and $y_{1}, z_{1}, w_{1}$ are all $3^{+}$-vertices. Suppose to the contrary that $|\mathcal{T}(v)| \geq 2$. Now, assume that there exist two bad paths $P$ and $P^{\prime}$ starting from $v y_{1}, v z_{1}$, respectively. We denote them by $P=v y_{1} \cdots y_{s}$ and $P^{\prime}=v z_{1} \cdots z_{t}$. Obviously, $d\left(y_{s}\right)=d\left(z_{t}\right)=2$ and the remaining internal vertices of $P$ and $P^{\prime}$ are all 3-vertices. Let $x_{1}^{\prime}$ denote the other neighbor of $x_{1}$ distinct from $v$. Let $y_{j}^{\prime}$ be the third neighbor of $y_{j}$ that is not on $P$. Similarly, let $z_{k}^{\prime}$ be the third neighbor of $z_{k}$ that is not on $P^{\prime}$. For our convenience, we denote $C_{1}=\left\{y_{1}, \cdots, y_{s}\right\}$ and $C_{2}=\left\{z_{1}, \cdots, z_{t}\right\}$. We only need to consider the two cases as follows.

Case $1 y z \notin E(G)$ for all $y \in C_{1}$ and $z \in C_{2}$

This implies that $C_{1} \cap C_{2}=\emptyset$. Let $\mathcal{B}=V(P) \cup V\left(P^{\prime}\right) \cup\left\{x_{1}\right\}$ and $H=G-\mathcal{B}$. Let $c$ denote an $L$-in-coloring of $H$ for its special orientation $\vec{H}$. To complete the proof of Case 1, we have to discuss the following two possibilities, depending on the situations of $x_{1}, y_{s}$ and $z_{t}$. 
Case 1.1 $x_{1} \neq y_{s} \neq z_{t} \neq x_{1}$.

We define an orientation for the edge set $E(G[\mathcal{B}])$ and those edges between $V(H)$ and $\mathcal{B}$, as shown in Fig. 6(1). The resulting orientation of $\vec{G}$ is also special. We can color $v, x_{1}, y_{1}, \cdots, y_{s}, z_{1}, \cdots, z_{t}$, successively, because

- $S(v)=N^{*}\left(w_{1}\right) \cup\left\{x_{1}^{\prime}, y_{1}^{\prime}, z_{1}^{\prime}\right\}$;

- $S\left(x_{1}\right)=N^{*}\left(x_{1}^{\prime}\right) \cup\left\{v, w_{1}\right\}$;

- $S\left(y_{1}\right)=N^{*}\left(y_{1}^{\prime}\right) \cup\left\{v, w_{1}, y_{2}^{\prime}\right\}$;

- $S\left(y_{2}\right)=N^{*}\left(y_{2}^{\prime}\right) \cup\left\{y_{1}, y_{1}^{\prime}, v, y_{3}^{\prime}\right\}$;

- $S\left(y_{j}\right)=N^{*}\left(y_{j}^{\prime}\right) \cup\left\{y_{j-1}, y_{j-2}, y_{j-1}^{\prime}, y_{j+1}^{\prime}\right\}$, for each $j \in\{3, \cdots, s-1\}$;

- $S\left(y_{s}\right)=N^{*}\left(y_{s}^{\prime}\right) \cup\left\{y_{s-1}, y_{s-1}^{\prime}, y_{s-2}\right\}$;

- $S\left(z_{1}\right)=N^{*}\left(z_{1}^{\prime}\right) \cup\left\{v, w_{1}, z_{2}^{\prime}\right\}$

- $S\left(z_{2}\right)=N^{*}\left(z_{2}^{\prime}\right) \cup\left\{z_{1}, z_{1}^{\prime}, v, z_{3}^{\prime}\right\}$;

- $S\left(z_{i}\right)=N^{*}\left(z_{i}^{\prime}\right) \cup\left\{z_{i-1}, z_{i-2}, z_{i-1}^{\prime}, z_{i+1}^{\prime}\right\}$, for each $i \in\{3, \cdots, t-1\}$;

- $S\left(z_{t}\right)=N^{*}\left(z_{t}^{\prime}\right) \cup\left\{z_{t-1}, z_{t-1}^{\prime}, z_{t-2}\right\}$.

Case 1.2 $x_{1}=y_{s} \neq z_{t}$.

We define an orientation for the edge set $E(G[\mathcal{B}])$ and those edges between $V(H)$ and $\mathcal{B}$, as depicted in Fig. 6(2). The resulting orientation of $\vec{G}$ is special. We can color $v, y_{1}, \cdots, y_{s}, z_{1}, \cdots, z_{t}$, successively, because

- $S(v)=N^{*}\left(w_{1}\right) \cup\left\{y_{1}^{\prime}, z_{1}^{\prime}\right\}$;

- $S\left(y_{1}\right)=N^{*}\left(y_{1}^{\prime}\right) \cup\left\{v, w_{1}, y_{2}^{\prime}\right\}$;

- $S\left(y_{2}\right)=N^{*}\left(y_{2}^{\prime}\right) \cup\left\{y_{1}, y_{1}^{\prime}, v, y_{3}^{\prime}\right\}$;

- $S\left(y_{j}\right)=N^{*}\left(y_{j}^{\prime}\right) \cup\left\{y_{j-1}, y_{j-2}, y_{j-1}^{\prime}, y_{j+1}^{\prime}\right\}$, for each $j \in\{3, \cdots, s-2\}$;

- $S\left(y_{s-1}\right)=N^{*}\left(y_{s-1}^{\prime}\right) \cup\left\{y_{s-2}, y_{s-2}^{\prime}, y_{s-3}, v\right\}$;

- $S\left(y_{s}\right)=\left\{v, w_{1}, y_{s-1}, y_{s-1}^{\prime}, y_{s-2}\right\}$;

- $S\left(z_{1}\right)=N^{*}\left(z_{1}^{\prime}\right) \cup\left\{v, w_{1}, z_{2}^{\prime}\right\}$

- $S\left(z_{2}\right)=N^{*}\left(z_{2}^{\prime}\right) \cup\left\{z_{1}, z_{1}^{\prime}, v, z_{3}^{\prime}\right\}$;

- $S\left(z_{i}\right)=N^{*}\left(z_{i}^{\prime}\right) \cup\left\{z_{i-1}, z_{i-2}, z_{i-1}^{\prime}, z_{i+1}^{\prime}\right\}$, for each $i \in\{3, \cdots, t-1\}$;

- $S\left(z_{t}\right)=N^{*}\left(z_{t}^{\prime}\right) \cup\left\{z_{t-1}, z_{t-1}^{\prime}, z_{t-2}\right\}$.

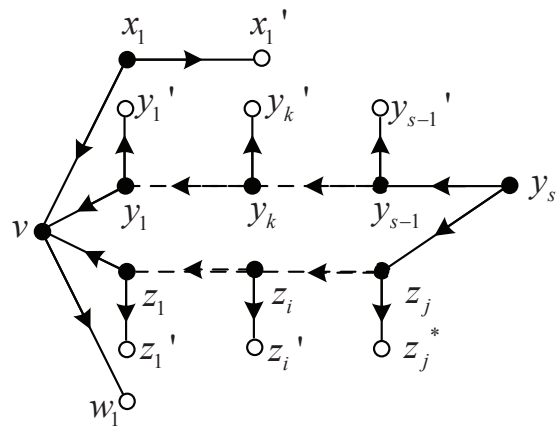

Fig. $6(3): z_{j} y_{s} \in E(G)$ and $x_{1} \neq y_{s}$. 
Case $2 y z \in E(G)$, where $y \in C_{1}$ and $z \in C_{2}$.

We need to consider the following two subcases, according to the situation of $z$.

Case 2.1 $z \in\left\{z_{1}, \cdots, z_{t-1}\right\}$.

We may denote $z=z_{j}$ such that $z_{j}$ is the 3 -vertex nearest to $v$ on $P^{\prime}$. The proof is divided into two possibilities.

Case 2.1.1 $y=y_{s}$.

This means that $z_{j} y_{s} \in E(G)$ for some fixed $j \in\{1, \cdots, t\}$. Let $\mathcal{B}=V(P) \cup\left\{x_{1}, z_{1}, \cdots, z_{j}\right\}$ and $H=G-\mathcal{B}$. Let $c$ denote an $L$-in-coloring of $H$ for its special orientation $\vec{H}$. We define an orientation for the edge set $E(G[\mathcal{B}])$ and those edges between $V(H)$ and $\mathcal{B}$, as shown in Fig. 6(3). The resulting orientation $\vec{G}$ is special. We can color $v, x_{1}, y_{1}, \cdots, y_{s-1}, z_{1}, \cdots, z_{j}, y_{s}$, successively, because

- $S(v)=N^{*}\left(w_{1}\right) \cup\left\{x_{1}^{\prime}, y_{1}^{\prime}, z_{1}^{\prime}\right\}$;

- $S\left(x_{1}\right)=N^{*}\left(x_{1}^{\prime}\right) \cup\left\{v, w_{1}\right\}$;

- $S\left(y_{1}\right)=N^{*}\left(y_{1}^{\prime}\right) \cup\left\{v, w_{1}, y_{2}^{\prime}\right\}$;

- $S\left(y_{2}\right)=N^{*}\left(y_{2}^{\prime}\right) \cup\left\{y_{1}, y_{1}^{\prime}, v, y_{3}^{\prime}\right\}$;

- $S\left(y_{k}\right)=N^{*}\left(y_{k}^{\prime}\right) \cup\left\{y_{k-1}, y_{k-2}, y_{k-1}^{\prime}, y_{k+1}^{\prime}\right\}$, for each $k \in\{3, \cdots, s-2\}$;

- $S\left(y_{s-1}\right)=N^{*}\left(y_{s-1}^{\prime}\right) \cup\left\{y_{s-2}, y_{s-2}^{\prime}, y_{s-3}\right\}$

- $S\left(z_{1}\right)=N^{*}\left(z_{1}^{\prime}\right) \cup\left\{v, w_{1}, z_{2}^{\prime}\right\}$;

- $S\left(z_{2}\right)=N^{*}\left(z_{2}^{\prime}\right) \cup\left\{z_{1}, z_{1}^{\prime}, v, z_{3}^{\prime}\right\}$;

- $S\left(z_{i}\right)=N^{*}\left(z_{i}^{\prime}\right) \cup\left\{z_{i-1}, z_{i-2}, z_{i-1}^{\prime}, z_{i+1}^{\prime}\right\}$, for each $i \in\{3, \cdots, j-2\}$;

- $S\left(z_{j-1}\right)=N^{*}\left(z_{j-1}^{\prime}\right) \cup\left\{z_{j-2}, z_{j-2}^{\prime}, z_{j-3}, z_{j}^{*}\right\}$;

- $S\left(z_{j}\right)=N^{*}\left(z_{j}^{*}\right) \cup\left\{z_{j-1}, z_{j-1}^{\prime}, z_{j-2}, y_{s-1}\right\}$;

- $S\left(y_{s}\right)=\left\{y_{s-1}, y_{s-1}^{\prime}, y_{s-2}, z_{j}, z_{j}^{*}, z_{j-1}\right\}$,

Case 2.1.2 $y \in\left\{y_{1}, \cdots, y_{s-1}\right\}$.

Without loss of generality, we may let $y=y_{k}$. If $z_{j} y_{s} \in E(G)$, then a good cycle $y_{s} y_{s-1} \cdots y_{k} z_{j} y_{s}$ is formed, contradicting Claim 4 If $z_{j} y_{l} \in E(G)$ for some fixed $l \in\{k+1, k+2, \cdots, s-1\}$, then a removable cycle $y_{l} y_{l-1} \cdots y_{k} z_{j} y_{l}$ with a heavy 3 -vertex $y_{l}$ is formed, contradicting Claim 5 . So, in what follows, we suppose that there is no edge connecting $z_{j}$ and one vertex belonging to $\left\{y_{k+1}, \cdots, y_{s}\right\}$. On the other hand, we recall that $z_{q}$ with $q \in\{1, \cdots, j-1\}$ is not adjacent to any vertex of $y_{k+1}, \cdots, y_{s}$ since $z_{j}$ is the nearest vertex to $v$ on $P^{\prime}$.

Let $\mathcal{B}=V(P) \cup\left\{x_{1}, z_{1}, \cdots, z_{j}\right\}$ and $H=G-\mathcal{B}$. Let $c$ denote an $L$-in-coloring of $H$ for its special orientation $\vec{H}$. We need to deal with the following two possibilities, according to the situations of $x_{1}$ and $y_{s}$.

(i) $x_{1} \neq y_{s}$.

We define an orientation for the edge set $E(G[\mathcal{B}])$ and those edges between $V(H)$ and $\mathcal{B}$, as depicted in Fig. 6(4). Clearly, the resulting orientation of $\vec{G}$ is special. We can color $v, x_{1}, z_{1}, \cdots, z_{j}, y_{1}, \cdots, y_{s}$, successively, because

- $S(v)=N^{*}\left(w_{1}\right) \cup\left\{x_{1}^{\prime}, y_{1}^{\prime}, z_{1}^{\prime}\right\}$

- $S\left(x_{1}\right)=N^{*}\left(x_{1}^{\prime}\right) \cup\left\{v, w_{1}\right\}$;

- $S\left(z_{1}\right)=N^{*}\left(z_{1}^{\prime}\right) \cup\left\{v, w_{1}, z_{2}^{\prime}\right\}$; 


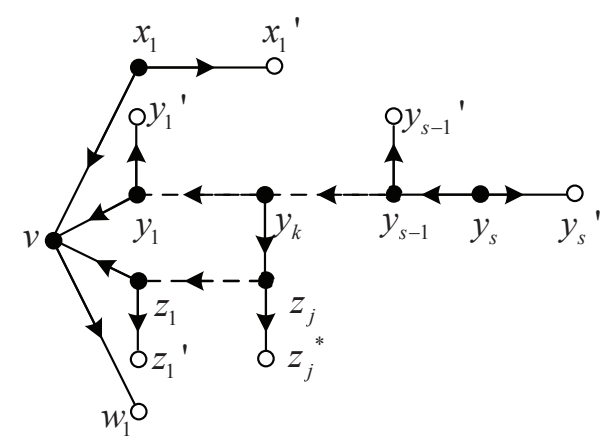

Fig. 6 (4): $z_{j} y_{k} \in E(G)$ and $x_{1} \neq y_{s}$.

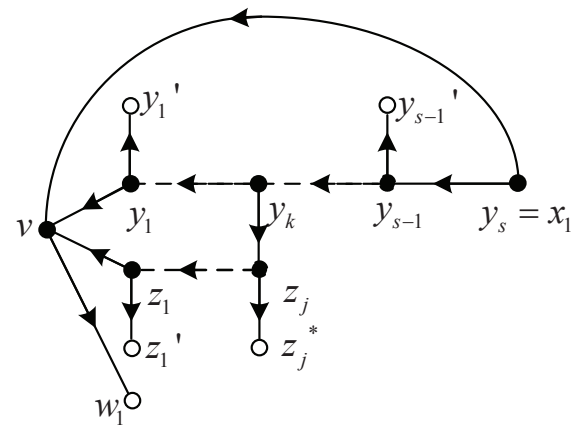

Fig. $6(5): z_{j} y_{k} \in E(G)$ and $x_{1}=y_{s}$.

- $S\left(z_{i}\right)=N^{*}\left(z_{i}^{\prime}\right) \cup\left\{z_{i-1}, z_{i-2}, z_{i-1}^{\prime}, z_{i+1}^{\prime}\right\}$, for each $i \in\{2, \cdots, j-2\}$;

- $S\left(z_{j-1}\right)=N^{*}\left(z_{j-1}^{\prime}\right) \cup\left\{z_{j-2}, z_{j-2}^{\prime}, z_{j-3}, z_{j}^{*}\right\}$;

- $S\left(z_{j}\right)=N^{*}\left(z_{j}^{*}\right) \cup\left\{z_{j-1}, z_{j-2}, z_{j-1}^{\prime}\right\}$

- $S\left(y_{1}\right)=N^{*}\left(y_{1}^{\prime}\right) \cup\left\{v, w_{1}, y_{2}^{\prime}\right\}$

- $S\left(y_{l}\right)=N^{*}\left(y_{l}^{\prime}\right) \cup\left\{y_{l-1}, y_{l-2}, y_{l-1}^{\prime}, y_{l+1}^{\prime}\right\}$, for each $l \in\{2, \cdots, k-2\}$;

- $S\left(y_{k-1}\right)=N^{*}\left(y_{k-1}^{\prime}\right) \cup\left\{y_{k-2}, y_{k-3}, y_{k-2}^{\prime}, z_{j}\right\}$

- $S\left(y_{k}\right)=\left\{y_{k-1}, y_{k-2}, y_{k-1}^{\prime}, z_{j}, z_{j-1}, z_{j}^{*}, y_{k+1}^{\prime}\right\}$

- $S\left(y_{k+1}\right)=N^{*}\left(y_{k+1}\right) \cup\left\{y_{k}, y_{k-1}, z_{j}, y_{k+2}^{\prime}\right\}$

- $S\left(y_{p}\right)=N^{*}\left(y_{p}^{\prime}\right) \cup\left\{y_{p-1}, y_{p-2}, y_{p-1}^{\prime}, y_{p+1}^{\prime}\right\}$ for each $p \in\{k+2, \cdots, s-1\}$;

- $S\left(y_{s}\right)=N^{*}\left(y_{s}^{\prime}\right) \cup\left\{y_{s-1}, y_{s-1}^{\prime}, y_{s-2}\right\}$.

(ii) $x_{1}=y_{s}$.

We define an orientation for the edge set $E(G[\mathcal{B}])$ and those edges in $V(H)$ and $\mathcal{B}$, as shown in Fig. 6(5).

The resulting orientation of $\vec{G}$ is special. We can color $v, z_{1}, \cdots, z_{j}, y_{1}, \cdots, y_{s}$, successively, because

- $S(v)=N^{*}\left(w_{1}\right) \cup\left\{y_{1}^{\prime}, z_{1}^{\prime}\right\}$;

- $S\left(z_{1}\right)=N^{*}\left(z_{1}^{\prime}\right) \cup\left\{v, w_{1}, z_{2}^{\prime}\right\}$

- $S\left(z_{i}\right)=N^{*}\left(z_{i}^{\prime}\right) \cup\left\{z_{i-1}, z_{i-2}, z_{i-1}^{\prime}, z_{i+1}^{\prime}\right\}$, for each $i \in\{2, \cdots, j-2\}$;

- $S\left(z_{j-1}\right)=N^{*}\left(z_{j-1}^{\prime}\right) \cup\left\{z_{j-2}, z_{j-2}^{\prime}, z_{j-3}, z_{j}^{*}\right\}$

- $S\left(z_{j}\right)=N^{*}\left(z_{j}^{*}\right) \cup\left\{z_{j-1}, z_{j-2}, z_{j-1}^{\prime}\right\}$;

- $S\left(y_{1}\right)=N^{*}\left(y_{1}^{\prime}\right) \cup\left\{v, w_{1}, y_{2}^{\prime}\right\}$;

- $S\left(y_{l}\right)=N^{*}\left(y_{l}^{\prime}\right) \cup\left\{y_{l-1}, y_{l-2}, y_{l-1}^{\prime}, y_{l+1}^{\prime}\right\}$, for each $l \in\{2, \cdots, k-2\}$;

- $S\left(y_{k-1}\right)=N^{*}\left(y_{k-1}^{\prime}\right) \cup\left\{y_{k-2}, y_{k-3}, y_{k-2}^{\prime}, z_{j}\right\}$;

- $S\left(y_{k}\right)=\left\{y_{k-1}, y_{k-2}, y_{k-1}^{\prime}, z_{j}, z_{j-1}, z_{j}^{*}, y_{k+1}^{\prime}\right\}$;

- $S\left(y_{k+1}\right)=N^{*}\left(y_{k+1}\right) \cup\left\{y_{k}, y_{k-1}, z_{j}, y_{k+2}^{\prime}\right\}$

- $S\left(y_{p}\right)=N^{*}\left(y_{p}^{\prime}\right) \cup\left\{y_{p-1}, y_{p-2}, y_{p-1}^{\prime}, y_{p+1}^{\prime}\right\}$ for each $p \in\{k+2, \cdots, s-2\}$;

- $S\left(y_{s-1}\right)=N^{*}\left(y_{s-1}^{\prime}\right) \cup\left\{y_{s-2}, y_{s-2}^{\prime}, y_{s-3}, v\right\}$;

- $S\left(y_{s}\right)=\left\{y_{s-1}, y_{s-1}^{\prime}, y_{s-2}, v, w_{1}\right\}$.

Case $2.2 z=z_{t}$.

The proof can be reduced to the previous Case 2.1.1. 
Therefore, we have completed the proof of Claim 8 .

Now we use a discharging argument with initial charge $\omega(v)=d(v)$ at each vertex $v$ and with the following two discharging rules (R1) and (R2). We write $\omega^{*}$ to denote the charge at each vertex $v$ after we apply the discharging rules. Note that the discharging rules do not change the sum of the charges. To complete the proof, we show that $\omega^{*}(v) \geq 3$ for all $v \in V(G)$. This leads to the following obvious contradiction:

$$
3 \leq \frac{\sum_{v \in V(G)} \omega^{*}(v)}{|V(G)|}=\frac{\sum_{v \in V(G)} \omega(v)}{|V(G)|}=\frac{2|E(G)|}{|V(G)|} \leq \operatorname{mad}(G)<3 .
$$

Hence no counterexample can exist.

Our discharging rules are defined as follows:

(R1) Each 2-vertex gets $\frac{1}{2}$ from each of its neighbors.

(R2) Each 3(1)-vertex gets $\frac{1}{4}$ from each of its sponsors.

Let us check that $\omega^{*}(v) \geq 3$ for each $v \in V(G)$. By Claim 1 , we derive that $\delta(G) \geq 2$. In the following argument, we let $v_{1}, v_{2}, \cdots, v_{d(v)}$ denote all neighbors of $v$ in a cyclic order. The following discussion is divided into five cases.

Case $1 d(v)=2$.

Then $\omega(v)=2$. By Claim 2, there is no 2(1)-vertex. It means that $v_{1}, v_{2}$ are both $3^{+}$-vertices. Therefore, $\omega^{*}(v) \geq 2+\frac{1}{2} \times 2=3$ by (R1).

Case $2 d(v)=3$.

Then $\omega(v)=3$. Recall that $\mathcal{S P}(v)$ denotes the set of sponsors of $v$, which was defined before Claim 6 We begin with the following claim.

Claim 9 If $v$ is a $3(1)$-vertex, then $|\mathcal{S P}(v)| \geq 2$.

Proof. Without loss of generality, suppose that $v_{1}$ is a 2 -vertex and $v_{2}, v_{3}$ are both $3^{+}$-vertices. By Claim 3 , it is easy to deduce that there exist at least two bad paths starting from $v v_{2}$ and $v v_{3}$, respectively. It follows immediately that $|\mathcal{S P}(v)| \geq 2$.

According to Claim 2, we infer that $v$ is neither a $3(2)$-vertex nor a $3(3)$-vertex. So, it suffices to consider the following two subcases.

- If $v$ is a $3(0)$-vertex, then $v$ sends nothing to each $v_{i}$ by $(\mathrm{R} 1)$ and $(\mathrm{R} 2)$ and thus $\omega^{*}(v)=3$.

- Now we suppose that $v$ is a 3(1)-vertex. Without loss of generality, assume that $d\left(v_{1}\right)=2$ and $d\left(v_{2}\right), d\left(v_{3}\right) \geq 3$. By (R1), $v$ sends a charge $\frac{1}{2}$ to $v_{1}$. On the other hand, by Claim 9 , we observe that $v$ has at least two sponsors, each of which sends a charge $\frac{1}{4}$ to $v$ by (R2). Therefore, $\omega^{*}(v) \geq 3-\frac{1}{2}+\frac{1}{4} \times 2=3$.

Case $3 d(v)=4$.

This implies that $\omega(v)=4$. By using Claim 2, we derive that $v$ is neither a $4(4)$-vertex nor a $4(3)$ vertex. If $v$ is a $4(2)$-vertex, then $|\mathcal{T}(v)|=0$ by Claim 7 So, $\omega^{*}(v) \geq 4-\frac{1}{2} \times 2=3$ by (R2). If $v$ is 
a $4(1)$-vertex, then $|\mathcal{T}(v)| \leq 1$ by Claim 8 and therefore $\omega^{*}(v) \geq 4-\frac{1}{2}-\frac{1}{4} \times 1=3 \frac{1}{4}>3$ by (R2). Finally, we suppose that $v$ is a $4(0)$-vertex. It means that $n_{2}(v)=0$. Then $|\mathcal{T}(v)| \leq 4$ by Claim 6 and we conclude that $\omega^{*}(v) \geq 4-\frac{1}{4} \times 4=3$ by (R2).

Case $4 d(v)=5$.

Obviously, $\omega(v)=5$. By Claim 2, we deduce that $n_{2}(v) \leq 3$. Moreover, it follows immediately from Claim 6 that $|\mathcal{T}(v)| \leq 5-n_{2}(v)$. So, by applying (R1) and (R2), we obtain that $\omega^{*}(v) \geq 5-\frac{1}{2} n_{2}(v)-$ $\frac{1}{4}|\mathcal{T}(v)| \geq 5-\frac{1}{2} n_{2}(v)-\frac{1}{4}\left(5-n_{2}(v)\right)=3 \frac{3}{4}-\frac{1}{4} n_{2}(v) \geq 3 \frac{3}{4}-\frac{1}{4} \times 3=3$.

Case $5 d(v) \geq 6$.

It follows directly from Claim 6 that $\omega^{*}(v) \geq d(v)-\frac{1}{2} n_{2}(v)-\frac{1}{4}|\mathcal{T}(v)| \geq d(v)-\frac{1}{2} n_{2}(v)-\frac{1}{4}(d(v)-$ $\left.n_{2}(v)\right)=\frac{3}{4} d(v)-\frac{1}{4} n_{2}(v) \geq \frac{3}{4} d(v)-\frac{1}{4} d(v)=\frac{1}{2} d(v) \geq 3$.

\section{Acknowledgements}

The authors would like to thank the referees for their valuable suggestions that helped to improve this work. The first author's research was supported by NSFC (No. 11101377). The third author's research was supported by NSFC (No. 11071223), ZJNSFC (No. Z6090150), ZJIP (No. T200905), ZJEDSRF (No. Y200805398), ZSDZZZZXK08 and IP-OCNS-ZJNU.

\section{References}

M. O. Albertson, G. G. Chappell, H. A. Kierstead, A. Kündgen, and R. Ramamurthi. Coloring with no 2-colored $p_{4}$ 's. Electron. J. Combin., 11(1):R26, 2004.

O. V. Borodin. On acyclic coloring of planar graphs. Discrete Math., 25:211-236, 1979.

Y. Bu, N. W. Cranston, M. Montassier, A. Raspaud, and W. Wang. Star-coloring of sparse graphs. J. Graph Theory, 62:201-219, 2009.

M. Chen, A. Raspaud, and W. Wang. Star list chromatic number of planar subcubic graphs. Submitted for publication, 2011.

G. Fertin, A. Raspaud, and B. Reed. On star-coloring of graphs. Lecture Notes in Comput. Sci., 2204:140-153, 2001.

B. Grünbaum. Acyclic colorings of planar graphs. Israel J. Math., 14:390-408, 1973.

H. A. Kierstead, A. Kündgen, and C. Timmons. Star coloring bipartite planar graphs. J. Graph Theory, 60:1-10, 2009.

A. V. Kostochka and L. S. Mel'nikov. Note to the paper of grünbaum on acyclic colorings. Discrete Math., 14: 403-406, 1976.

A. Kündgen and C. Timmons. Star coloring planar graphs from small lists. J. Graph Theory, 63(4):324-337, 2010.

J. Nešetřil and P. Ossana de Mendez. Colorings and homomorphisms of minor closed classes. 17:651-664, 2003.

M. Tarsi. On the decomposition of a graph into stars. Discrete Math., 36:299-304, 1981.

C. Timmons. Star-coloring planar graphs. Master's Thesis, California State University San Marcos, 2007. 